\title{
Quantum Fisher information and coherence in one-dimensional $X Y$ spin models with Dzyaloshinsky-Moriya interactions
}

\author{
Biao-Liang $\mathrm{Ye}^{1,2^{*}}, \mathrm{Bo} \mathrm{Li}^{3,4}$, Zhi-Xi Wang ${ }^{5}$, Xianqing Li-Jost ${ }^{4,6}$, and Shao-Ming Fei ${ }^{4,5 *}$ \\ ${ }^{1}$ Quantum Information Research Center, School of Physics and Electronic Information, Shangrao Normal University, Shangrao 334001, China; \\ ${ }^{2}$ Jiangxi Province Key Laboratory of Polymer Preparation and Processing, Shangrao 334001, China; \\ ${ }^{3}$ School of Mathematics and Computer Sciences, Shangrao Normal University, Shangrao 334001, China; \\ ${ }^{4}$ Max-Planck-Institute for Mathematics in the Sciences, Leipzig 04103, Germany; \\ ${ }^{5}$ School of Mathematical Sciences, Capital Normal University, Beijing 100048, China; \\ ${ }^{6}$ School of Mathematics and Statistics, Hainan Normal University, Haikou 571158, China
}

Received March 25, 2018; accepted June 13, 2018; published online August 8, 2018

\begin{abstract}
We investigate quantum phase transitions in $X Y$ spin models using Dzyaloshinsky-Moriya (DM) interactions. We identify the quantum critical points via quantum Fisher information and quantum coherence, finding that higher DM couplings suppress quantum phase transitions. However, quantum coherence (characterized by the $l_{1}$-norm and relative entropy) decreases as the DM coupling increases. Herein, we present both analytical and numerical results.
\end{abstract}

quantum Fisher information, quantum coherence, $X Y$ spin models, Dzyaloshinsky-Moriya interactions

PACS number(s): $\quad$ 03.67.-a, 64.70.Tg, 75.10.Pq

Citation: B.-L. Ye, B. Li, Z.-X. Wang, X. Li-Jost, and S.-M. Fei, Quantum Fisher information and coherence in one-dimensional XY spin models with Dzyaloshinsky-Moriya interactions, Sci. China-Phys. Mech. Astron. 61, 110312 (2018), https://doi.org/10.1007/s11433-018-9262-9

\section{Introduction}

Quantum entanglement plays important roles in quantum physics and quantum information processing [1-9], such as in quantum key distribution [2,3], quantum secure direct communication [4-7], quantum machine learning [8]. Quantum phase transition, a key element of condensed matter physics, is a type of quantum fluctuation that occurs in spin-chain systems at zero temperature. Understanding the connections between quantum entanglement and critical behavior near a quantum phase transition is especially important [10-12].

In recent decades, additional quantum measures beyond entanglement have been proposed [13-17]. Quantum phase transitions have been intensively studied using correlation measures $[18,19]$. In particular, spin-chain systems,

*Corresponding authors (Biao-Liang Ye, email: biaoliangye@gmail.com; Shao-Ming Fei, email: feishm@cnu.edu.cn) including the $X Y, X X Z$, and LMG models, have been used to investigate quantum discord [20-24], one-norm quantum discord [25], and quantum deficit [26], among others [27-32]. Recently, quantum coherence has attracted considerable interest as a resource. The $l_{1}$ norm and relative entropy of coherence are very intuitive and coherence quantifiers are easy to compute and fully monotone in all possible coherence resource theories [33].

Quantum Fisher information (QFI) [34] is a central quantity in quantum metrology [35], where a linear interferometer is used to estimate the unitary dynamics [29]. QFI also plays a significant role in quantum detection and estimation as it provides a bound on the quantum estimation accuracy. In addition, QFI signaling of quantum phase transitions has received much attention [36].

The $X Y$ model with Dzyaloshinsky-Moriya (DM) inter- 
actions has been extensively investigated. However, only a few studies have considered this model from the perspective of understanding the role played by quantum correlations in phase transitions [20,32,37]. In ref. [20], quantum phase transitions of the above mentioned model have also been investigated in terms of quantum concurrence, quantum discord, and classical correlations. Herein, we study the quantum phase transition of the $X Y$ model with DM interaction using QFI, in which QFI is an intrinsic and ubiquitous quantity that plays significant roles in quantum metrology, such as in parameter estimation. We also consider the $l_{1}$ norm and relative entropy of coherence.

\section{Preliminaries}

First, let us briefly review QFI and quantum coherence.

Quantum Fisher information. In general phase estimation scenarios, the evolution of a quantum state, given by the density matrix $\varrho$, under a unitary transformation can be described as $\varrho_{\theta}=\mathrm{e}^{-\mathrm{i} A \theta} \varrho \mathrm{e}^{\mathrm{i} A \theta}$, where $\theta$ is the phase shift and $A$ is an operator. The estimation accuracy for $\theta$ is limited by the quantum Cramér-Rao inequality [38,39]:

$\Delta \hat{\theta} \geq \frac{1}{\sqrt{v \mathcal{F}\left(\varrho_{\theta}\right)}}$,

where $\hat{\theta}$ denotes the unbiased estimator for $\theta, v$ is the number of times the measurement is repeated, and $\mathcal{F}\left(\varrho_{\theta}\right)$ is the so-called QFI.

QFI is defined as:

$\mathcal{F}(\varrho, A)=2 \sum_{m, n} \frac{\left(p_{m}-p_{n}\right)^{2}}{\left(p_{m}+p_{n}\right)}|\langle m|A| n\rangle|^{2}$,

where $p_{m}$ and $|m\rangle$ are the eigenvalues and eigenvectors, respectively, of the density matrix $\varrho$, which is used as a probe state to estimate $\theta$.

Quantum coherence. Quantum coherence is an important resource in quantum information tasks [40]. A rigorous theory has been proposed to determine a good indicator for measuring quantum coherence [33]. Herein, we focus on the $l_{1}$ norm and relative entropy of coherence.

(i) $l_{1}$ norm. The $l_{1}$ norm of coherence is defined as the sum of the absolute values of all off-diagonal elements in the density matrix $\varrho$ :

$C_{l_{1}}(\varrho)=\sum_{i \neq j}\left|\varrho_{i j}\right|$.

(ii) Relative entropy. The relative entropy of coherence (REC) is defined as:

$C_{\mathrm{REC}}(\varrho)=S\left(\varrho_{\mathrm{diag}}\right)-S(\varrho)$.
Here, $\varrho_{\text {diag }}$ is the diagonal part of $\varrho$, and the function $S(\sigma)=$ $-\operatorname{Tr} \sigma \log _{2} \sigma$ is the von Neumann entropy of the density matrix $\sigma$. In the following discussion, we will use both coherence measures as informational tools to study quantum phase transitions in the $X Y$ model with DM interactions.

\section{The $X Y$ model with DM interactions}

The Hamiltonian of the Heisenberg $X Y$ chain with DM interactions under periodic boundary conditions in the presence of an external field is described as follows:

$$
\begin{aligned}
H= & \sum_{i=1}^{N}\left\{J \left[(1+\gamma) \sigma_{i}^{x} \sigma_{i+1}^{x}+(1-\gamma) \sigma_{i}^{y} \sigma_{i+1}^{y}\right.\right. \\
& \left.\left.+D\left(\sigma_{i}^{x} \sigma_{i+1}^{y}-\sigma_{i}^{y} \sigma_{i+1}^{x}\right)\right]-\sigma_{i}^{z}\right\},
\end{aligned}
$$

where $\sigma_{i}^{\alpha}(\alpha=x, y, z)$ are the Pauli operators at the $i$ th lattice site, $N$ is the total number of spins, and $J$ is the inverse of the external transverse magnetic field strength. The system is antiferromagnetic when $J>0$ and ferromagnetic when $J<0$. The anisotropy $\gamma$ lies in the interval $[-1,1]$, with the values $\gamma=0$ and \pm 1 corresponding to the $X X$ and Ising models, respectively. Finally the factor $D$ represents the strength of the antisymmetric DM interaction along the $z$ direction. In the following, we consider the case $N \rightarrow \infty$ at zero temperature.

The $X Y$ model has been solved exactly, and the correlation functions were obtained from $[41,42]$. The $X Y$ model with DM interactions can be solved exactly using the JordanWigner transformation [43], as well [20]. The nonclassical correlation between two spins at sites $i$ and $j$ can be derived from their collective state, and the model can be reduced to the two-spin density matrix $\varrho(i, j)=\operatorname{Tr}_{\backslash i j} \varrho$, by tracing over all spins except those at sites $i$ and $j$. The Hamiltonian's translational invariance ensures that the density matrix $\varrho(i, j)=\varrho(i, i+r)$, where $r$ is the distance between the spins. This two-spin reduced density matrix exclusively depends on $r$ and is independent of the spins' actual locations. Thus, owing to the $U(1)$ invariance and symmetries of the Hamiltonian eq. (5), the model's two-spin density matrix can be written as:

$\varrho(i, i+r)=\left(\begin{array}{cccc}a_{+} & 0 & 0 & c_{-} \\ 0 & b & c_{+} & 0 \\ 0 & c_{+} & b & 0 \\ c_{-} & 0 & 0 & a_{-}\end{array}\right)$.

The entries in this density matrix are given by two-point correlation functions,

$a_{ \pm}=\frac{1}{4} \pm \frac{\left\langle\sigma_{i}^{z}\right\rangle}{2}+\frac{\left\langle\sigma_{i}^{z} \sigma_{i+r}^{z}\right\rangle}{4}$,

$b=\frac{1-\left\langle\sigma_{i}^{z} \sigma_{i+r}^{z}\right\rangle}{4}$,

$c_{ \pm}=\frac{\left\langle\sigma_{i}^{x} \sigma_{i+r}^{x}\right\rangle \pm\left\langle\sigma_{i}^{y} \sigma_{i+r}^{y}\right\rangle}{4}$. 
The model's magnetization in the presence of an external field is given by

$\left\langle\sigma_{i}^{z}\right\rangle=-\frac{1}{\pi} \int_{0}^{\pi} \mathrm{d} \phi \frac{J(\cos \phi-2 D \sin \phi)-1}{\Delta}$,

where

$\Delta=\sqrt{[J(\cos \phi-2 D \sin \phi)-1]^{2}+J^{2} \gamma^{2} \sin ^{2} \phi}$.

The two-point spin-spin correlation functions corresponding to the $x$ and $y$ directions can be computed from the determinants of the Toeplitz matrices, and the one corresponding to the $z$ direction can be computed from the magnetization, yielding

$\begin{aligned}\left\langle\sigma_{i}^{x} \sigma_{i+r}^{x}\right\rangle & =\left|\begin{array}{cccc}Q_{-1} & Q_{-2} & \ldots & Q_{-r} \\ Q_{0} & Q_{-1} & \ldots & Q_{-r+1} \\ \vdots & \vdots & \ddots & \vdots \\ Q_{r-2} & Q_{r-3} & \ldots & Q_{-1}\end{array}\right|, \\ \left\langle\sigma_{i}^{y} \sigma_{i+r}^{y}\right\rangle & =\left|\begin{array}{cccc}Q_{1} & Q_{0} & \ldots & Q_{-r+2} \\ Q_{2} & Q_{1} & \ldots & Q_{-r+3} \\ \vdots & \vdots & \ddots & \vdots \\ Q_{r} & Q_{r-1} & \ldots & Q_{1}\end{array}\right|,\end{aligned}$

and

$\left\langle\sigma_{i}^{z} \sigma_{i+r}^{z}\right\rangle=\frac{\left\langle\sigma^{z}\right\rangle^{2}-Q_{r} Q_{-r}}{4}$,

where

$$
\begin{aligned}
Q_{r}= & -\frac{1}{\pi} \int_{0}^{\pi} \mathrm{d} \phi \frac{2 \cos (\phi \cdot r)}{\Delta}[J(\cos \phi-2 D \sin \phi)-1] \\
& +\frac{\gamma}{\pi} \int_{0}^{\pi} \mathrm{d} \phi \frac{2 J \sin (\phi \cdot r)}{\Delta} \sin \phi .
\end{aligned}
$$

Therefore, the model's QFI and quantum coherence can be computed from the two-spin reduced density matrix eq. (6).

\section{Quantum Fisher information and quantum coherence for the $X Y$ model with DM interac- tions}

\subsection{Quantum Fisher information and quantum coher- ence}

Now, we study QFI for the $X Y$ model with DM interactions. Suppose $\varrho$ is an arbitrary bipartite state, whereas $\left\{A_{\mu}\right\}$ and $\left\{B_{\mu}\right\}$ are arbitrary complete sets of local orthonormal observables of the two subsystems with respect to $\varrho$. The QFI can then be written as [44]:

$\mathcal{F}=\sum_{\mu} \mathcal{F}\left(\varrho, A_{\mu} \otimes I+I \otimes B_{\mu}\right)$,

which is also the global information for $\varrho$. It has been proved that the value of QFI $\mathcal{F}$ given by eq. (14) is independent of the choice of local orthonormal bases [44], meaning that it is an intrinsic quantity of the composite system. For a general two-spin system, the local orthonormal observables $\left\{A_{\mu}\right\}$ and $\left\{B_{\mu}\right\}$ can be defined as:

$\left\{A_{\mu}\right\}=\left\{B_{\mu}\right\}=\frac{1}{\sqrt{2}}\left\{I, \sigma^{x}, \sigma^{y}, \sigma^{z}\right\}$.

Consequently, until $\varrho$ is given, QFI $\mathcal{F}$ can be calculated from eq. (2).

For an $X Y$ chain with DM interactions, after tedious computation we can obtain the QFI for the two-spin state eq. (6):

$$
\begin{aligned}
\mathcal{F}= & \frac{\left(\left\langle\sigma_{i}^{x} \sigma_{i+r}^{x}\right\rangle-\left\langle\sigma_{i}^{y} \sigma_{i+r}^{y}\right\rangle\right)^{2}}{1+\left\langle\sigma_{i}^{z} \sigma_{i+r}^{z}\right\rangle}+\left[\left(3\left\langle\sigma_{i}^{z}\right\rangle^{2}+\left\langle\sigma_{i}^{z} \sigma_{i+r}^{z}\right\rangle^{2}-2\left\langle\sigma_{i}^{z} \sigma_{i+r}^{z}\right\rangle\right)\left(\left\langle\sigma_{i}^{x} \sigma_{i+r}^{x}\right\rangle+\left\langle\sigma_{i}^{y} \sigma_{i+r}^{y}\right\rangle\right)\right. \\
& +\left(1-2\left\langle\sigma_{i}^{z} \sigma_{i+r}^{z}\right\rangle\right)\left(\left\langle\sigma_{i}^{x} \sigma_{i+r}^{x}\right\rangle^{2}+\left\langle\sigma_{i}^{y} \sigma_{i+r}^{y}\right\rangle^{2}\right)+2\left(\left\langle\sigma_{i}^{z}\right\rangle^{2}+\left\langle\sigma_{i}^{z} \sigma_{i+r}^{z}\right\rangle^{2}-2\left\langle\sigma_{i}^{z}\right\rangle^{2}\left\langle\sigma_{i}^{z} \sigma_{i+r}^{z}\right\rangle\right) \\
& \left.+\left(\left\langle\sigma_{i}^{x} \sigma_{i+r}^{x}\right\rangle^{3}+\left\langle\sigma_{i}^{y} \sigma_{i+r}^{y}\right\rangle^{3}\right)\right] /\left[\left(1+\left\langle\sigma_{i}^{x} \sigma_{i+r}^{x}\right\rangle\right)\left(1+\left\langle\sigma_{i}^{y} \sigma_{i+r}^{y}\right\rangle\right)-\left\langle\sigma_{i}^{z}\right\rangle^{2}\right] .
\end{aligned}
$$

Next, we compute the model's coherence. As the two-spin reduced density matrix eq. (6) was computed in the $\sigma_{z}$ basis, we study the coherence in the same basis. Calculating the $l_{1}$ norm is straightforward:

$C_{l_{1}}=\left|\left\langle\sigma_{i}^{x} \sigma_{i+r}^{x}\right\rangle\right|$.
Using the relative entropy formula eq. (4), we have

$C_{\mathrm{REC}}=\sum_{k=0}^{1}\left(\xi_{k} \log \xi_{k}+\eta_{k} \log \eta_{k}-\zeta_{k} \log \zeta_{k}\right)-2 \varepsilon \log \varepsilon$,

where

$\xi_{k}=\left[1-\left\langle\sigma_{i}^{z} \sigma_{i+r}^{z}\right\rangle+(-1)^{k}\left(\left\langle\sigma_{i}^{x} \sigma_{i+r}^{x}\right\rangle+\left\langle\sigma_{i}^{y} \sigma_{i+r}^{y}\right\rangle\right)\right] / 4$, 


$$
\begin{aligned}
\eta_{k}= & {\left[(-1)^{k} \sqrt{4\left\langle\sigma_{i}^{z}\right\rangle^{2}+\left(\left\langle\sigma_{i}^{x} \sigma_{i+r}^{x}\right\rangle-\left\langle\sigma_{i}^{y} \sigma_{i+r}^{y}\right\rangle\right)^{2}}\right.} \\
& \left.+1+\left\langle\sigma_{i}^{z} \sigma_{i+r}^{z}\right\rangle\right] / 4, \\
\zeta_{k}= & {\left[1+\left\langle\sigma_{i}^{z} \sigma_{i+r}^{z}\right\rangle+(-1)^{k} 2\left\langle\sigma_{i}^{z}\right\rangle\right] / 4, }
\end{aligned}
$$

and

$\varepsilon=\left[1-\left\langle\sigma_{i}^{z} \sigma_{i+r}^{z}\right\rangle\right] / 4$.

\subsection{Quantum phase transitions}

Next, we show how quantum phase transitions can be detected in the $X Y$ model with DM interactions. Figure 1 plots the QFI, $l_{1}$ norm of coherence, and REC for $X Y$ chains with different DM interaction values $D$, namely $0,0.5$, and 1 . These show that the QFI in the $J<1$ region differs from that in the $J>1$ region, and it changes substantially at $D=0$ for $J>1$ (first column). However, the QFI becomes stable as $D$ increases (see $D=0.5,1$ ). We can also observe that the coherences increase with $\gamma$ and reach maximal values at $\gamma=1$ for $J>1$, with the critical point occurring at $J=1$. The two coherence measures, namely the $l_{1}$ norm and REC, behave similarly and form clear layers for different values of $D$. For example, in Figure 1(ii), we can see that as $D$ becomes larger, the coherence becomes smaller, indicating that higher $D$ values suppress quantum coherence. All plots show clear differences between the two regions $J \in[0,1]$ and $J \in[1,2]$. The model's quantum phase transition occurs at the critical point $J=1$. We can also see the same behavior for the nextnearest $(r=2)$ and fifth-nearest $(r=5)$ neighbor cases.

We now investigate the $X X(\gamma=0)$ and Ising $(\gamma=1)$ models with DM interactions, as shown in Figure 2 for the relationships between the QFI, $l_{1}$ norm of coherence, and REC vs. the parameters $J$ and $D$. In each subfigure, the upper and lower surfaces represent the nearest $(r=1)$ and fifth-nearest $(r=5)$ neighbor spins, respectively. For the $X X$ model, we observe critical behavior at $J=1$. The QFI decreases as $J$ increases in the $J>1$ region, but the coherence increases. The behaviors for the fifth-nearest and nearest neighbor spin cases are identical. For the Ising model, the QFI decreases as $J$ increases in the nearest neighbor case. However, it exhibits different behavior in the fifth-nearest neighbor case, where the QFI either decreases to a minimal value or increases to a finite value. The coherences increase with $J$.

Figure 3 shows the QFI, $l_{1}$ norm of coherence, and REC as functions of $J$ for the nearest neighbor $(r=1)$ spins in the transverse Ising model $(\gamma=1)$ with different DM interaction parameters $D$. The first row shows the QFI, $l_{1}$ norm, and REC vs. $J$, whereas the second row shows the corresponding derivative coincidences. The blue, red, and green lines correspond to DM parameters $D=0,0.5,1$, respectively. Here,

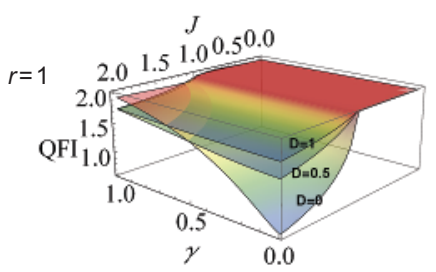

(i)

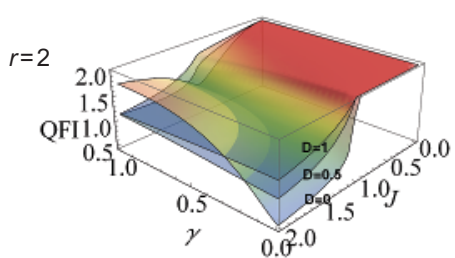

(iv)

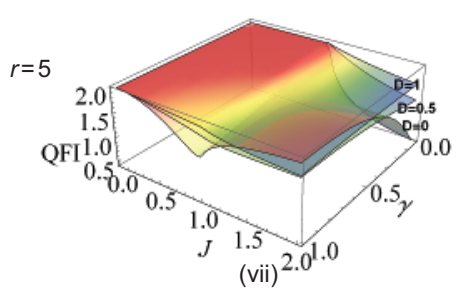

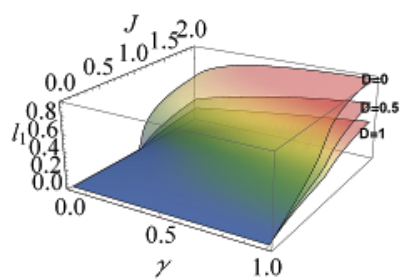

(ii)

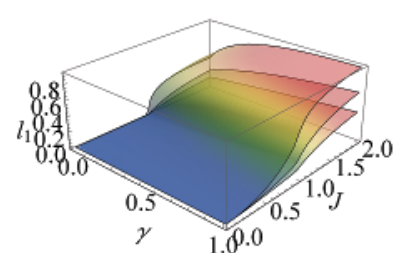

(v)

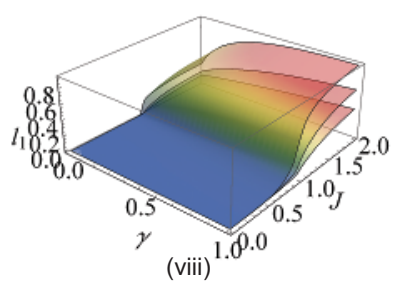

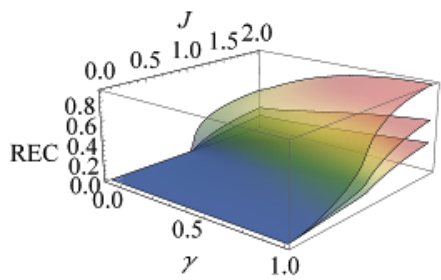

(iii)

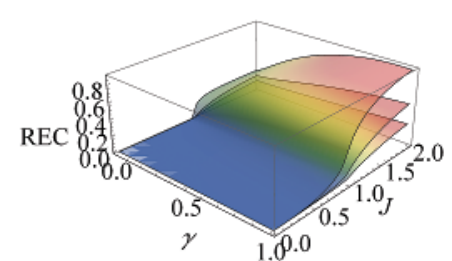

(vi)

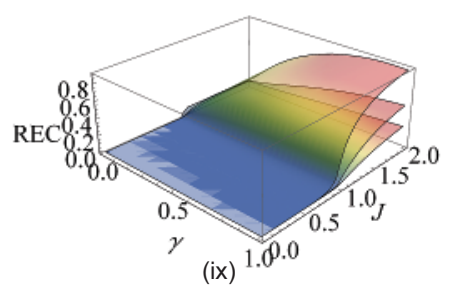

Figure 1 (Color online) QFI, $l_{1}$ norm of coherence and REC for the $X Y$ model with DM interactions vs. $\gamma$ and $J$, for the DM parameter $D=0,0.5,1$. The first (i,ii,iii), second (iv,v,vi), and third (vii,viii,ix) rows correspond to the nearest $(r=1)$, next-nearest $(r=2)$, and fifth-nearest ( $r=5$ ) neighbors, respectively. The first (i,iv,vii), second (ii,v,viii) and third (iii,vi,ix) columns correspond to the quantum Fisher information (QFI), $l_{1}$ norm of coherence, and relative entropy of coherence (REC), respectively. The DM interaction parameter $D$ is $D=0,0.5,1$ for the top, middle and bottom surfaces, respectively, in Figure (ii), (iii), (v), (vi), (viii), and (ix). 
we notice that, similar to the QFI behavior, the coherences have cusps at $J=1$. In addition, they are clearly suppressed as $D$ increases, and their derivatives clearly show nonanalytic behavior at the critical points, implying that a quantum phase transition occurs at $J=1$.

Providing a contrast to Figures 3 and 4 shows the fifthnearest neighbor $(r=5)$ spin in the $\gamma=1 / 2$ model. Here, when $D=0$ the QFI decreases for $J \leq 1$ and increases for $J>1$. However, the QFI exhibits different behavior when $D=0.5$ and 1 , for which it decreases and has a cusp at $J=1$. In addition, its derivative is negative when $D=0.5,1$, and positive when $D=0$. A quantum phase transition can be seen clearly at $J=1$. The coherence is initially zero, then increases quickly near $J=1$ before saturating at a finite value. The derivative of the QFI and coherence exhibit nonanalytic behavior at the critical point $J=1$, and the DM parameter suppresses quantum phase transition.
Figure 5 shows the derivatives of the QFI, $l_{1}$ norm of coherence, and REC with respect to the DM interaction parameter $D$. The dashed, solid, and dot-dashed lines correspond to the QFI, $l_{1}$ norm, and REC respectively, whereas the blue, orange, green, and red lines correspond to $J=0.9,1.0,1.2,2.0$, respectively. Here, the derivatives show distinct behaviors for different $J$ regions. The QFI and $l_{1}$ norm derivatives are always negative, but the coherence derivatives (dot-dashed lines) are positive, except for the low $D$ region when $J=2$. No nonanalytic behavior is observed, as was found for quantum discord [20].

\section{Conclusions}

In this paper, we have studied quantum phase transitions in anisotropic $X Y$ chains with DM interactions using QFI and
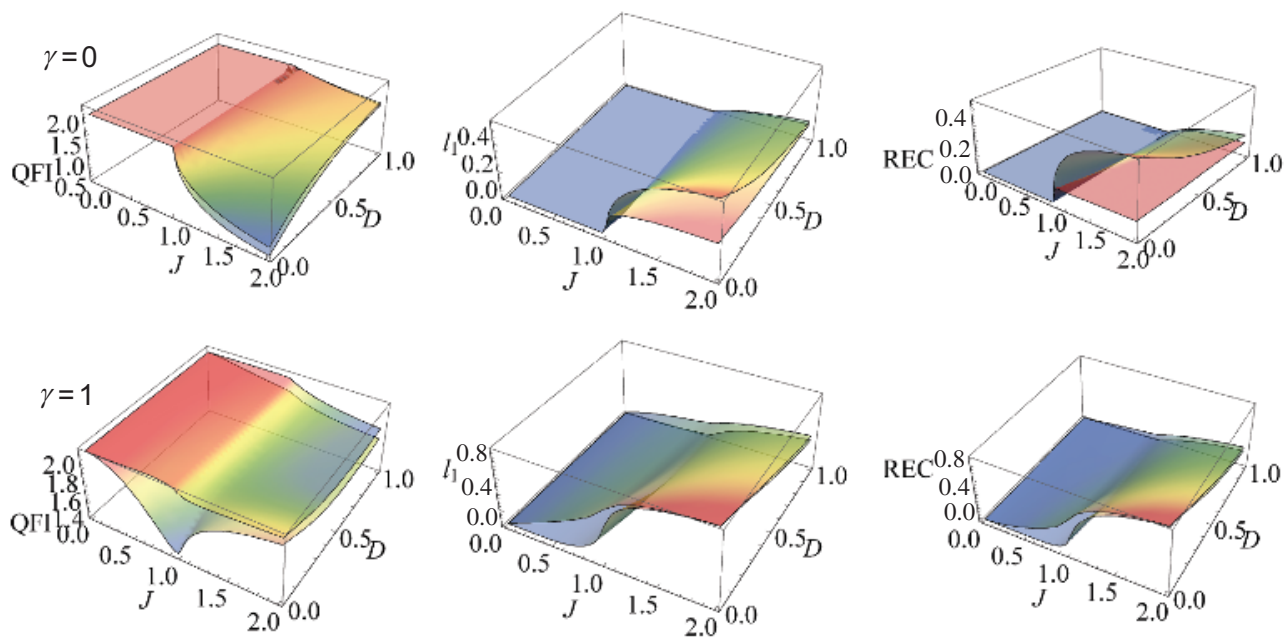

Figure 2 (Color online) QFI, $l_{1}$ norm of coherence, and REC vs. $J$ and $D$ for the $X X(\gamma=0)$ (first row) and Ising ( $\left.\gamma=1\right)$ (second row) models. In all subfigures, the upper and lower surfaces correspond to the nearest $(r=1)$ and fifth-nearest $(r=5)$ neighbor cases, respectively.
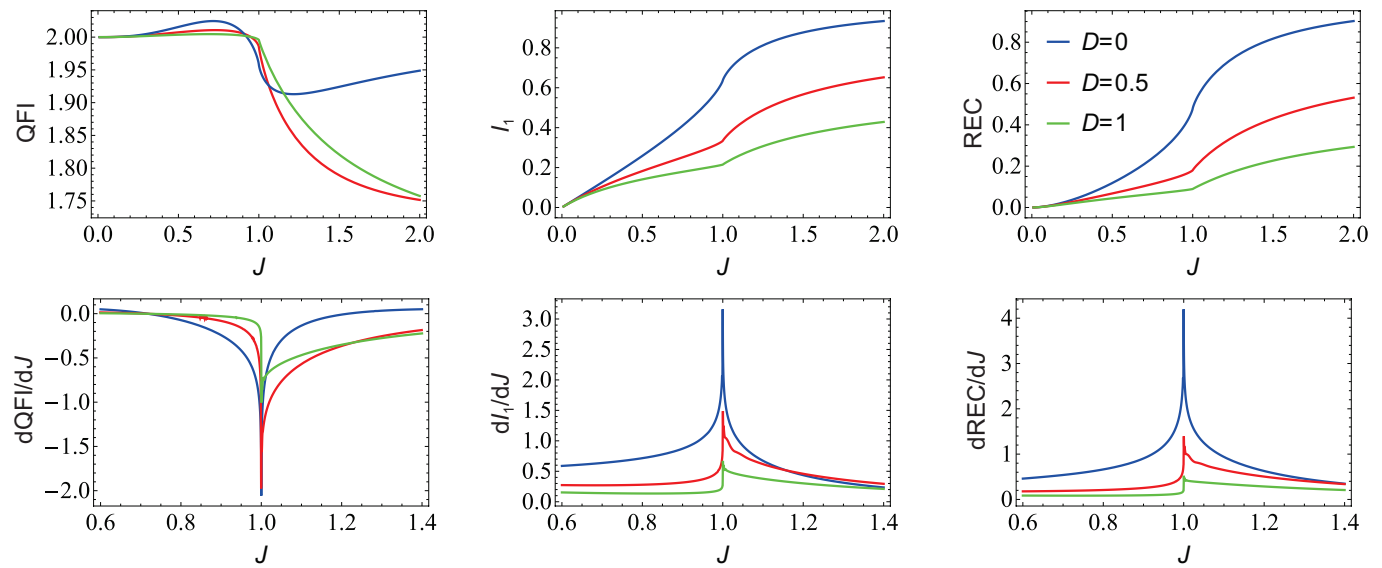

Figure 3 (Color online) QFI, $l_{1}$ norm of coherence, REC, and their derivatives vs. $J$ for the nearest neighbor $(r=1)$ spins in the $\gamma=1 X Y$ model with DM interactions. The blue, red, and green lines correspond to $D=0,0.5,1$, respectively. 

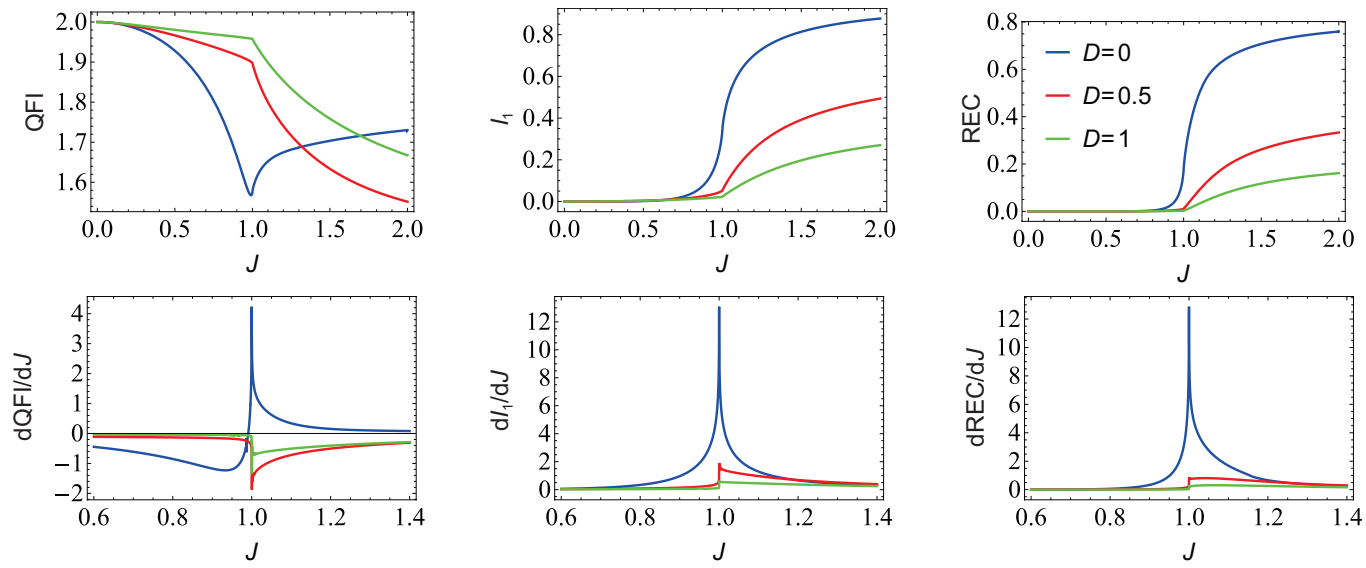

Figure 4 (Color online) QFI, $l_{1}$ norm of coherence, REC, and their derivatives vs. $J$ for the fifth-nearest neighbor $(r=5)$ spins in the $\gamma=1 / 2 X Y$ model with DM interactions, for $D=0,0.5,1$.

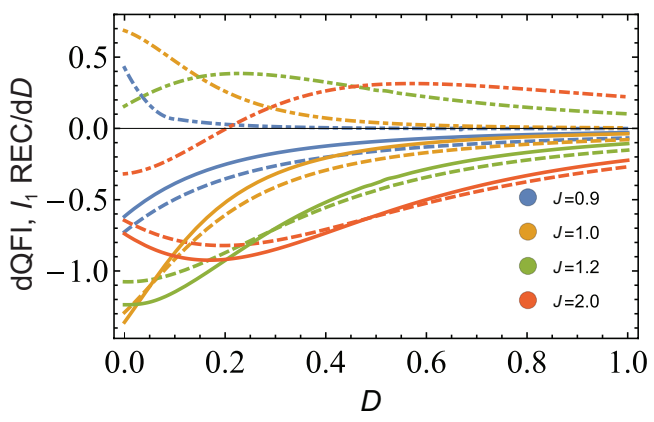

Figure 5 (Color online) First derivatives of QFI, $l_{1}$ norm of coherence, and REC with respect to $D$ for nearest neighbor spins $(r=1)$ in the $\gamma=1 / 2 X Y$ model with DM interactions. The dashed, solid, and dot-dashed lines correspond to the QFI, $l_{1}$ norm, and REC respectively, with blue, orange, green, and red lines denoting $J=0.9,1.0,1.2,2.0$, respectively.

quantum coherence. In particular, we have identified quantum critical points using QFI and quantum coherence. We have also shown that the coherence (characterized by the $l_{1}$ norm and relative entropy) decreases as the DM interaction increases, and that the DM coupling suppresses the quantum phase transition. These results improve our understanding of the relationships between the QFI, quantum coherence, and quantum phase transitions in spin systems. These quantities are important when investigating quantum phase transitions in realistic experimental scenarios. This approach could also be used to explore quantum phase transitions in other spinchain systems.

This work was supported by the National Natural Science Foundation of China (Grant Nos. 11675113, and 11765016), the Natural Science Foundation of Beijing (Grant No. KZ201810028042), and Jiangxi Education Department Fund (Grant Nos. GJJ161056, and KJLD14088). Open access funding provided by Max Planck Society.

Open Access This article is distributed under the terms of the Creative Commons Attribution 4.0 International License (http://creativecommons.org/licenses/by/4.0/), which permits use, duplication, adaptation, distribution and reproduction in any medium or format, as long as you give appropriate credit to the original author(s) and the source, provide a link to the Creative Commons license and indicate if changes were made.

1 R. Horodecki, P. Horodecki, M. Horodecki, and K. Horodecki, Rev. Mod. Phys. 81, 865 (2009).

2 A. K. Ekert, Phys. Rev. Lett. 67, 661 (1991).

3 G. L. Long, and X. S. Liu, Phys. Rev. A 65, 032302 (2002).

4 F. G. Deng, G. L. Long, and X. S. Liu, Phys. Rev. A 68, 042317 (2003).

5 W. Zhang, D. S. Ding, Y. B. Sheng, L. Zhou, B. S. Shi, and G. C. Guo, Phys. Rev. Lett. 118, 220501 (2017), arXiv: 1609.09184.

6 F. Zhu, W. Zhang, Y. Sheng, and Y. Huang, Sci. Bull. 62, 1519 (2017).

7 F. Z. Wu, G. J. Yang, H. B. Wang, J. Xiong, F. Alzahrani, A. Hobiny, and F. G. Deng, Sci. China-Phys. Mech. Astron. 60, 120313 (2017).

8 Y. B. Sheng, and L. Zhou, Sci. Bull. 62, 1025 (2017).

9 C. M. Xie, Y. M. Liu, J. L. Chen, X. F. Yin, and Z. J. Zhang, Sci. China-Phys. Mech. Astron. 59, 100314 (2016).

10 A. Osterloh, L. Amico, G. Falci, and R. Fazio, Nature 416, 608 (2002).

11 T. J. Osborne, and M. A. Nielsen, Phys. Rev. A 66, 032110 (2002).

12 G. Vidal, J. I. Latorre, E. Rico, and A. Kitaev, Phys. Rev. Lett. 90, 227902 (2003).

13 H. Ollivier, and W. H. Zurek, Phys. Rev. Lett. 88, 017901 (2001).

14 L. Henderson, and V. Vedral, J. Phys. A-Math. Gen. 34, 6899 (2001).

15 J. Oppenheim, M. Horodecki, P. Horodecki, and R. Horodecki, Phys. Rev. Lett. 89, 180402 (2002).

16 S. Luo, Phys. Rev. A 77, 022301 (2008).

17 K. Modi, T. Paterek, W. Son, V. Vedral, and M. Williamson, Phys. Rev. Lett. 104, 080501 (2010), arXiv: 0911.5417.

18 K. Modi, A. Brodutch, H. Cable, T. Paterek, and V. Vedral, Rev. Mod. Phys. 84, 1655 (2012), arXiv: 1112.6238.

19 G. Adesso, T. R. Bromley, and M. Cianciaruso, J. Phys. A-Math. Theor. 49, 473001 (2016), arXiv: 1605.00806.

20 B. Q. Liu, B. Shao, J. G. Li, J. Zou, and L. A. Wu, Phys. Rev. A 83, 052112 (2011), arXiv: 1012.2788.

21 R. Dillenschneider, Phys. Rev. B 78, 224413 (2008), arXiv: 0809.1723.

22 M. S. Sarandy, Phys. Rev. A 80, 022108 (2009), arXiv: 0905.1347.

23 T. Werlang, C. Trippe, G. A. P. Ribeiro, and G. Rigolin, Phys. Rev. 
Lett. 105, 095702 (2010), arXiv: 1006.3332.

24 S. Campbell, J. Richens, N. L. Gullo, and T. Busch, Phys. Rev. A 88, 062305 (2013), arXiv: 1309.1052.

25 J. D. Montealegre, F. M. Paula, A. Saguia, and M. S. Sarandy, Phys. Rev. A 87, 042115 (2013), arXiv: 1303.5110.

26 B. L. Ye, B. Li, L. J. Zhao, H. J. Zhang, and S. M. Fei, Sci. China-Phys. Mech. Astron. 60, 030311 (2017), arXiv: 1702.03123.

27 G. Karpat, B. Çakmak, and F. F. Fanchini, Phys. Rev. B 90, 104431 (2014), arXiv: 1404.6427.

28 A. Misra, A. Biswas, A. K. Pati, A. Sen(De), and U. Sen, Phys. Rev. E 91, 052125 (2015), arXiv: 1406.5065.

29 Ł. Czekaj, A. Przysiężna, M. Horodecki, and P. Horodecki, Phys. Rev. A 92, 062303 (2015), arXiv: 1403.5867.

30 Y. C. Li, and H. Q. Lin, Sci. Rep. 6, 26365 (2016).

31 A. L. Malvezzi, G. Karpat, B. Çakmak, F. F. Fanchini, T. Debarba, and R. O. Vianna, Phys. Rev. B 93, 184428 (2016), arXiv: 1602.03731.

32 C. Radhakrishnan, I. Ermakov, and T. Byrnes, Phys. Rev. A 96, 012341 (2017), arXiv: 1707.03545.

33 T. Baumgratz, M. Cramer, and M. B. Plenio, Phys. Rev. Lett. 113,
140401 (2014), arXiv: 1311.0275.

34 S. L. Braunstein, and C. M. Caves, Phys. Rev. Lett. 72, 3439 (1994).

35 V. Giovannetti, S. Lloyd, and L. Maccone, Phys. Rev. Lett. 96, 010401 (2006).

36 X. M. Liu, W. W. Cheng, and J. M. Liu, Sci. Rep. 6, 19359 (2016).

37 F. Altintas, and R. Eryigit, Ann. Phys. 327, 3084 (2012), arXiv: 1202.1495 .

38 C. W. Helstrom, Quantum Detection and Estimation Theory (Academic Press, New York, 1976).

39 A. S. Holevo, Probabilistic and Statistical Aspects of Quantum Theory (North-Holland, Amsterdam, 1982).

40 A. Streltsov, G. Adesso, and M. B. Plenio, Rev. Mod. Phys. 89, 041003 (2017), arXiv: 1609.02439.

41 E. Lieb, T. Schultz, and D. Mattis, Ann. Phys. 16, 407 (1961).

42 J. H. H. Perk, and H. W. Capel, Physica A 89, 265 (1977).

43 T. Giamarchi, Quantum Physics in One Dimension (Oxford University, New York, 2004).

44 N. Li, and S. Luo, Phys. Rev. A 88, 014301 (2013). 